\title{
La mortalité par blessure non intentionnelle et ses causes externes au Canada entre 2001 et 2007
}

\author{
Y. Chen, Ph. D. (1); F. Mo, Ph. D. (2); Q. L. Yi, Ph. D. (1); Y. Jiang, M. Sc. (2); Y. Mao, Ph. D. (2)
}

Cet article a fait l'objet d'une évaluation par les pairs.

\section{Résumé}

Introduction : Une bonne compréhension des caractéristiques et des tendances temporelles de la mortalité par blessure non intentionnelle est cruciale pour l'élaboration de stratégies de prévention.

Méthodologie : Nous avons analysé les statistiques de l'état civil au Canada (à l'exclusion du Québec) entre 2001 et 2007. Les taux de mortalité ont été normalisés selon l'âge et le sexe par rapport à la population canadienne de 2001. Un modèle autorégressif a été utilisé pour l'analyse de séries chronologiques.

Résultats : Le taux global de mortalité a diminué régulièrement, alors que le taux de mortalité par blessure non intentionnelle est resté stable durant la période étudiée. Les taux les plus élevés ont été observés dans les trois territoires. Les décès par blessure non intentionnelle étaient moins fréquents chez les enfants que chez les jeunes ou les adultes. Après 60 ans, la mortalité augmentait de façon soutenue avec l'âge. Les hommes étaient plus nombreux à décéder des suites d'une blessure non intentionnelle, et le ratio hommes-femmes culminait dans le groupe d'âge des 25 à 29 ans. Les collisions de véhicules à moteur, les chutes et les empoisonnements étaient les trois causes principales. On a observé une augmentation constante et marquée de la mortalité attribuable aux chutes. Les décès attribuables aux collisions de véhicules à moteur et aux noyades étaient plus fréquents durant les mois d'été, tandis que ceux causés par les chutes et les brûlures étaient plus fréquents durant les mois d'hiver.

Conclusion : La part des blessures non intentionnelles dans l'ensemble des causes de décès et la mortalité attribuable aux chutes ont augmenté au Canada entre 2001 et 2007.

Mots-clés : normalisation selon l'âge, brûlure, Canada, produit de consommation, noyade, chute, mortalité, empoisonnement, blessure non intentionnelle, suffocation, collision de véhicules à moteur, statistiques de l'état civil

\section{Introduction}

Les blessures figurent parmi les principales causes de décès et d'incapacité dans le monde ${ }^{1}$. Elles représentent quelque $16 \%$ de la charge mondiale de morbidité ${ }^{2}$ et sont la principale cause de décès chez les personnes âgées de moins de $60 \mathrm{ans}^{3}$. En 2004, l'Organisation mondiale de la Santé a estimé que les blessures étaient à l'origine de plus de 5 millions de décès par an, dont 3,9 millions étaient consécutifs à des blessures non intentionnelles ${ }^{4}$. Comparativement à de nombreuses maladies, les blessures touchent plus souvent les jeunes et, par conséquent, engendrent un plus grand nombre d'années de vie perdues $^{1}$. Au Canada, en 2004, le fardeau économique total des blessures était d'environ 20 milliards de dollars, dont
16 milliards de dollars imputables à des blessures non intentionnelles ${ }^{5}$.

Une blessure non intentionnelle est une blessure qui n'est pas infligée volontairement ou avec l'intention de blesser. Étant donné que toutes les blessures non intentionnelles ne sont pas des événements aléatoires et que certaines peuvent être prévenues, il n'est pas approprié d'utiliser systématiquement le terme " accident " pour les définir. Les blessures non intentionnelles peuvent être subdivisées en plusieurs catégories en fonction de leurs causes externes : collisions de véhicules à moteur, chutes, empoisonnements, noyades, suffocation, etc. ${ }^{6}$. Les blessures non intentionnelles peuvent être liées au travail ou aux loisirs. Les différents types de blessures non intentionnelles peuvent avoir des profils spécifiques selon certaines sous-populations : les accidents de la circulation routière sont le plus fréquents chez les jeunes ${ }^{7}$, tandis que les chutes sont plus susceptibles d'avoir une issue fatale chez les personnes âgées ${ }^{8}$. La surveillance de l'évolution des tendances concernant la mortalité par blessure non intentionnelle (mortalité globale et mortalité selon la cause) permet de recueillir une information essentielle à l'élaboration de nouveaux programmes de prévention et d'intervention dans ce domaine et l'adaptation de ceux déjà existant. Pour cette étude, nous avons procédé à une analyse descriptive des données statistiques de l'état civil afin d'étudier les caractéristiques et les tendances temporelles de la mortalité par blessure non intentionnelle - mortalité globale et selon la cause - au Canada (à l'exclusion du Québec).

Rattachement des auteurs :

1. Département d'épidémiologie et de médecine sociale, Université d'Ottawa, Ottawa (Ontario), Canada

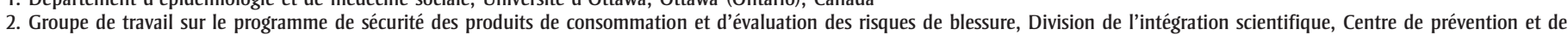
contrôle des maladies chroniques, Agence de la santé publique du Canada, Ottawa (Ontario), Canada

Correspondance : Yue Chen, Département d'épidémiologie et de médecine sociale, Faculté de médecine, Université d’Ottawa, 451, chemin Smyth, Ottawa (Ontario) K1H 8M5; tél. : 613 562-5800, poste 8287; téléc. : 613 562-5465; courriel : ychen@uottawa.ca 


\section{Méthodologie}

L'étude a fait appel aux données statistiques de l'état civil du Canada contenues dans la Base de données sur les décès (à l'exclusion des décès enregistrés dans la province de Québec, puisque ces données n'étaient pas disponibles dans le Système d'extraction et d'analyse des données de l'Agence de la santé publique du Canada) et portant sur la période du $1^{\mathrm{er}}$ janvier 2001 au 31 décembre 2007. Elles reposent sur les données des certificats de décès et sont transmises à Statistique Canada par les bureaux provinciaux et territoriaux de l'état civil. Les données sur les décès utilisées dans cette analyse sont codées conformément au système de la Classification internationale des maladies, $10^{e}$ révision (CIM-10), où les causes externes sont classées en différentes catégories identifiées par des codes alphanumériques (V01 à Y98). Ces codes ont été utilisés pour identifier les décès par blessure non intentionnelle (CIM-10 : V01 à X59, Y85 et Y86) et les décès par blessure non intentionnelle selon la cause : collisions de véhicules à moteur (V02 à V04[.9], V09.2, V12 à V14[.3-.9], V19[.4-.6], V20 à V28[.3-.9], V29[.4-.9], V30 à V79[.4-.9], V80[.3-.5], V81 à V82[.1], V83 à V86[.0.3], V87[.0-.8], V89.2); bicyclettes (CIM10 : V10 à V14, V16 à V19); piétons (en lien avec la circulation routière) (CIM-10 : V02 à V04[.1], V09.2, V09.3); navigation de plaisance (CIM-10 : V90.2 à V90.8, V91.2 à V91.8, V92.2 à V92.8, V93.2 à V93.8, V94.2 à V94.8); noyades (CIM-10 : V90, V92, W65 à W74); chutes (CIM-10 : W00 à W19); brûlures ou feux (CIM-10 : W85 à W91, X00 à X19); suffocation (CIM10 : W75 à W84); empoisonnement (CIM10 : X40 à X49) ainsi que d'autres causes non intentionnelles.

Nous nous sommes servis des estimations de la population provenant des statistiques démographiques annuelles de Statistique Canada ${ }^{9,10}$ pour calculer les taux de mortalité normalisés selon l'âge et le sexe, en utilisant la méthode directe, avec comme référence la population canadienne de 2001. Pour calculer les taux moyens de mortalité globale, nous avons, pour chaque province et pour les trois territoires combinés (Territoires du Nord-Ouest, Yukon et Nunavut), divisé le nombre total de décès durant cette période de 7 ans par la somme des populations annuelles, ce qui équivaut à une moyenne pondérée des taux annuels en utilisant comme poids la population annuelle.

Pour explorer les tendances temporelles des taux de mortalité par blessure non intentionnelle entre 2001 et 2007 ainsi que les variations saisonnières des décès par blessure non intentionnelle selon la cause chez les hommes et les femmes, nous avons effectué une analyse de séries chronologiques. Pour calculer les taux de mortalité, et à défaut d'estimation mensuelle de la population, nous avons utilisé comme dénominateur la population annuelle moyenne. Comme le nombre de décès par mois était peu élevé et ne permettait pas de stratification selon l'âge, nous avons procédé à une normalisation selon l'âge et le sexe en divisant le taux de mortalité mensuel normalisé par le taux de mortalité annuel brut (taux mensuel brut $\times$ taux normalisé/taux annuel brut). Nous avons ensuite effectué un tracé graphique des taux mensuels ajustés afin de présenter visuellement leurs tendances temporelles et leurs variations saisonnières. Nous avons créé des modèles autorégressifs ajustés afin de cerner les associations entre le mois, l'année civile et les différents décès par blessure non intentionnelle. Dans les modèles, nous avons pris en considération l'autocorrélation d'ordre 1, utilisant les taux mensuels comme variables dépendantes et les indicateurs de mois et d'année comme variables indépendantes.

Toutes les analyses ont été réalisées à l'aide du logiciel de statistiques SAS, version 9.1 (SAS Institute Inc., Cary, Caroline du Nord, États-Unis).

\section{Résultats}

Nous avons recensé au total 51178 décès attribuables à des blessures non intentionnelles durant la période étudiée, soit entre 2001 et 2007. Ces décès représentaient $4,2 \%$ de tous les décès au Canada à l'exclusion du Québec. Le taux de mortalité toutes causes confondues normalisé selon l'âge et le sexe a décliné régulièrement, passant de 702 pour
100000 en 2001 à 631 pour 100000 en 2007 ( $p<0,001)$, tandis que le taux de mortalité par blessure non intentionnelle est resté relativement stable d'une année à l'autre $(p=0,571)$. Cela signifie que l'importance relative de la mortalité par blessure non intentionnelle par rapport à la mortalité toutes causes confondues a augmenté de manière significative $(p=0,003)$ au cours de la période. Les hommes représentaient $61,1 \%$ de tous les décès par blessure non intentionnelle. Les tendances temporelles étaient toutefois similaires chez les hommes et les femmes, aussi bien en ce qui concerne la mortalité par blessure non intentionnelle que la mortalité globale (toutes causes confondues) et le ratio entre les deux (tableau 1).

Après normalisation selon l'âge et le sexe, c'est dans les trois territoires combinés que nous avons observé les taux de mortalité les plus élevés, à la fois pour la mortalité globale (842,3 pour 100 000) et pour la mortalité par blessure non intentionnelle (69,1 pour 100 000) (tableau 2). C'est la Colombie-Britannique qui affichait le plus faible taux de mortalité globale $(626,8$ pour 100 000), tandis que TerreNeuve-et-Labrador présentait le plus faible taux de mortalité par blessure non intentionnelle $(24,8$ pour 100 000). Le taux de mortalité par blessure non intentionnelle des trois territoires s'élevait à près du triple de celui de Terre-Neuve-etLabrador. L'Ontario avait un faible taux de mortalité par blessure non intentionnelle (26,3 pour 100 000), comparable à celui de Terre-Neuve-et-Labrador, mais la différence entre ces deux provinces était importante pour le taux de mortalité toutes causes confondues : 655,9 pour 100000 en Ontario et 802,9 pour 100000 à Terre-Neuve-et-Labrador. Les différences entre les autres provinces étaient relativement faibles, tant pour la mortalité toutes causes confondues que pour la mortalité par blessure non intentionnelle (tableau 2). Le tableau 2 montre également que, dans toutes les provinces et tous les territoires, le taux de mortalité par blessure non intentionnelle était plus élevé chez les hommes que chez les femmes, ce qui était également le cas pour le ratio taux de mortalité par blessure 
TABLEAU 1

Taux brut de mortalité et taux normalisé de mortalité, par année civile et selon le sexe, Canada (à l'exclusion du Québec), 2001-2007

\begin{tabular}{|c|c|c|c|c|c|}
\hline \multirow{2}{*}{$\begin{array}{l}\text { Année } \\
\text { civile }\end{array}$} & \multicolumn{2}{|c|}{ Taux brut de mortalité } & \multicolumn{3}{|c|}{ Taux normalisé de mortalitéa } \\
\hline & $\begin{array}{l}\text { Toutes causes } \\
\text { (pour } 100 \text { 000) }\end{array}$ & $\begin{array}{c}\text { Blessure non } \\
\text { intentionnelle } \\
\text { (pour } 100 \text { 000) }\end{array}$ & $\begin{array}{l}\text { Toutes causes } \\
\text { (pour } 100 \text { 000) }\end{array}$ & $\begin{array}{c}\text { Blessure non } \\
\text { intentionnelle } \\
\text { (pour } 100000 \text { ) }\end{array}$ & $\begin{array}{c}\text { Blessure non } \\
\text { intentionnelle / } \\
\text { Toutes causes (\%) }\end{array}$ \\
\hline \multicolumn{6}{|c|}{ Ensemble } \\
\hline 2001 & 701,8 & 28,7 & 702,1 & 28,7 & 4,09 \\
\hline 2002 & 704,3 & 29,9 & 692,3 & 29,6 & 4,28 \\
\hline 2003 & 710,3 & 29,5 & 685,3 & 28,8 & 4,20 \\
\hline 2004 & 702,2 & 28,7 & 664,6 & 27,7 & 4,27 \\
\hline 2005 & 708,5 & 30,1 & 656,8 & 28,6 & 4,35 \\
\hline 2006 & 699,2 & 30,5 & 633,6 & 28,5 & 4,50 \\
\hline 2007 & 709,7 & 32,0 & 631,0 & 29,7 & 4,71 \\
\hline \multicolumn{6}{|c|}{ Hommes } \\
\hline 2001 & 725,0 & 36,4 & 879,5 & 39,9 & 4,54 \\
\hline 2002 & 720,3 & 37,1 & 857,7 & 40,6 & 4,73 \\
\hline 2003 & 729,5 & 36,2 & 851,2 & 39,3 & 4,62 \\
\hline 2004 & 718,7 & 35,2 & 824,1 & 37,8 & 4,59 \\
\hline 2005 & 722,6 & 37,3 & 811,2 & 39,6 & 4,88 \\
\hline 2006 & 715,1 & 36,8 & 783,2 & 38,5 & 4,92 \\
\hline 2007 & 725,9 & 39,0 & 778,3 & 40,4 & 5,19 \\
\hline \multicolumn{6}{|c|}{ Femmes } \\
\hline 2001 & 679,1 & 21,2 & 572,7 & 18,5 & 3,23 \\
\hline 2002 & 688,5 & 22,8 & 571,3 & 19,7 & 3,45 \\
\hline 2003 & 691,4 & 22,9 & 564,3 & 19,3 & 3,42 \\
\hline 2004 & 686,0 & 22,4 & 547,8 & 18,4 & 3,36 \\
\hline 2005 & 694,5 & 23,0 & 650,3 & 18,9 & 2,91 \\
\hline 2006 & 683,5 & 24,3 & 626,4 & 19,4 & 3,10 \\
\hline 2007 & 693,8 & 25,0 & 624,7 & 20,0 & 3,20 \\
\hline
\end{tabular}

${ }^{a}$ Normalisé par rapport à l'ensemble de la population canadienne de 2001.

non intentionnelle sur taux de mortalité toutes causes confondues.

Chez les enfants, les décès par blessure non intentionnelle ont été moins nombreux (moins de 7 pour 100 000) que chez les jeunes et les adultes (tableau 3). Pour ces derniers (15-59 ans), les taux de mortalité par blessure non intentionnelle sont comparables entre les différentes tranches d'âge (variant entre 28,5 et 37,7 pour 100000 chez les hommes et entre 8,5 et 12,7 pour 100000 chez les femmes). Après 60 ans, le taux de mortalité augmente de façon constante avec l'âge, passant chez les hommes de 35,3 pour 100000 pour les 60 à 64 ans à 801,0 pour 100000 pour les plus de 90 ans, et chez les femmes de 14,4 à 663,1 pour 100000 . Dans tous les groupes d'âge, les hommes ont été plus nombreux à mourir des suites d'une blessure non intentionnelle (tableau 3). Le ratio de mortalité hommesfemmes augmente avec l'âge à partir du groupe des 5 à 9 ans $(1,34)$, culminant dans le groupe de 25 à 29 ans $(3,76)$ pour diminuer régulièrement ensuite. Le tableau 3 montre également que, parmi les décès par blessure non intentionnelle dont la cause a été déterminée, ce furent les collisions de véhicules à moteur qui ont été les plus répandues chez les hommes (taux de mortalité de 10,2 pour 100 000), suivies des chutes $(7,7$ pour 100 000) et des empoisonnements $(5,1$ pour 100 000). Chez les femmes, ce furent dans l'ordre les chutes, les collisions de véhicules à moteur et les empoisonnements, avec des taux de mortalité respectifs de 7,9, 4,5 et 2,2 pour 100000 .
Les taux de mortalité par blessure non intentionnelle selon une cause déterminée ont été dans tous les cas plus élevés chez les hommes que chez les femmes, sauf pour les chutes (tableau 3). Globalement, les décès attribuables aux chutes ont représenté $26 \%$ de tous les décès par blessure non intentionnelle, ceux attribuables à des collisions de véhicules à moteur $24 \%$ et les empoisonnements $12 \%$ (figure 1).

Même si le taux de mortalité par blessure non intentionnelle augmente de façon rapide après l'âge de 60 ans, il représente une fraction beaucoup plus élevée des décès toutes causes confondues dans les groupes d'âge plus jeunes, culminant dans le groupe des 15 à 19 ans (45,2\%), tant chez les hommes $(46,7 \%)$ que chez les femmes (41,8\%), avant de diminuer graduellement (tableau 4).

Nous avons analysé séparément les principales causes externes de décès par blessure non intentionnelle, à savoir les collisions de véhicules à moteur, les chutes, les empoisonnements, les accidents touchant un piéton (en lien avec la circulation routière), les noyades, les brûlures et les suffocations, et ce, en distinguant les hommes (tableau 5 et figure 2) et les femmes (tableau 6 et figure 3). Nous avons constaté une augmentation importante d'une année sur l'autre de la mortalité par blessure attribuable à une chute, tant chez les hommes $(p<0,01)$ que chez les femmes $(p<0,01)$. Pour les autres types de blessures, le taux de mortalité normalisé selon l'âge a diminué légèrement (pour les brûlures et les noyades chez les hommes, et pour les collisions de véhicules à moteur et les brûlures chez les femmes) ou n'a affiché aucun changement significatif. Pendant les mois d'été, le risque de décès par collision de véhicules à moteur et par noyade a été significativement plus élevé, avec une variation plus marquée chez les hommes. Pendant les mois d'hiver, les décès consécutifs à des chutes ou à des brûlures ont été plus fréquents. Comparativement aux mois de janvier-février, les décès par empoisonnement ont été relativement plus nombreux en mars-avril $(p<0,05)$, ceux par accident touchant un piéton en 
TABLEAU 2

Taux moyen de mortalité, par province/territoire et selon le sexe, Canada (à l'exclusion du Québec), 2001-2007

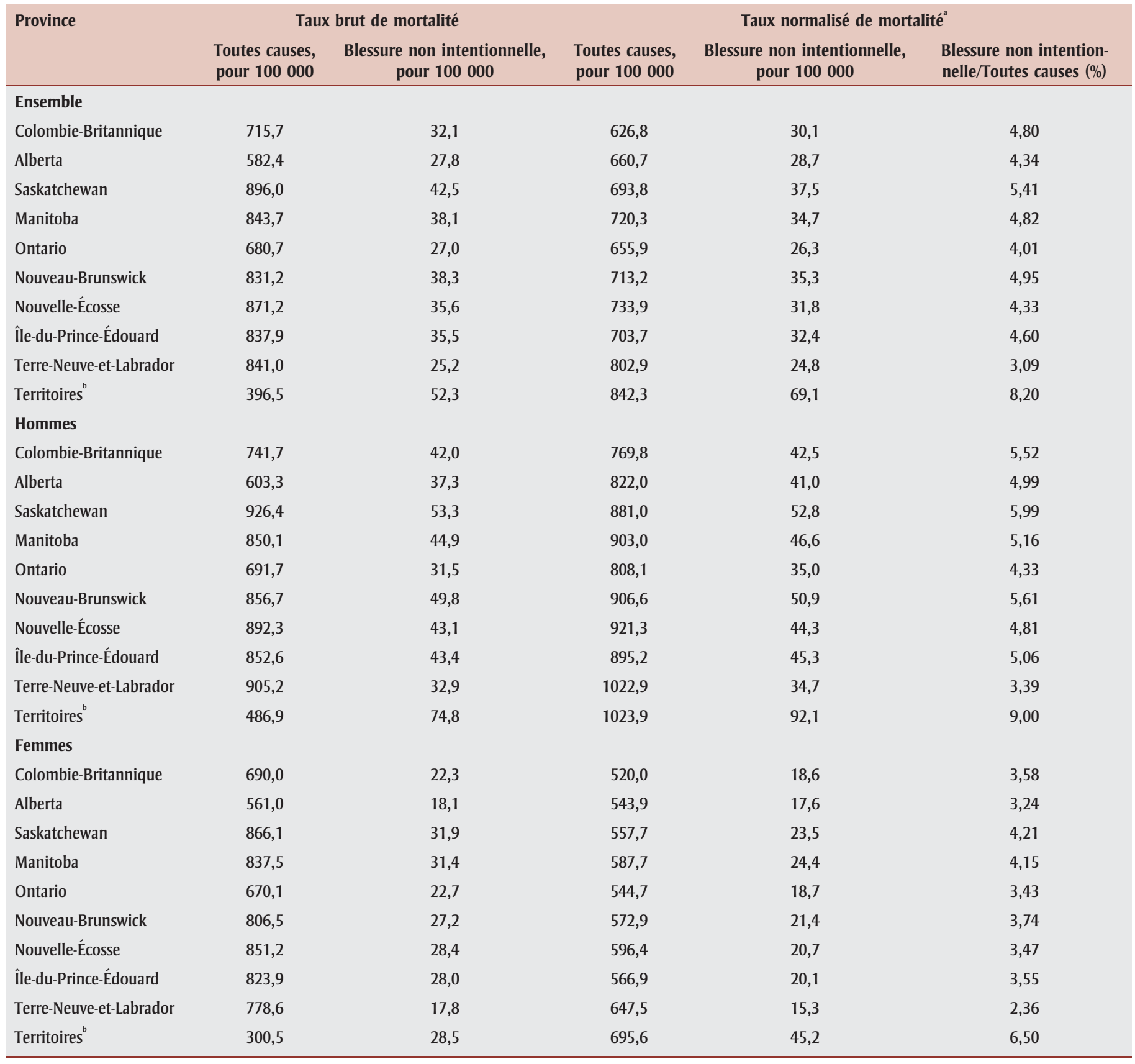

a Normalisé par rapport à l'ensemble de la population canadienne de 2001.

b Yukon, Territoires du Nord-Ouest, Nunavut.

septembre-octobre $(p<0,05)$ et en novembre-décembre $(p<0,01)$. Aucune différence significative n'a été notée entre les saisons dans le cas des décès par suffocation.

\section{Analyse}

Le taux de mortalité attribuable à une blessure non intentionnelle normalisé selon l'âge et le sexe est resté stable durant la période d'étude (2001-2007), tandis que le taux de mortalité toutes causes confondues a diminué d'environ $10 \%$. La part des décès par blessure non intentionnelle dans l'ensemble des décès toutes causes confondues est passée de 4,1 à 4,7 \%. Ces données révèlent une tendance à la hausse de la proportion des blessures non intentionnelles dans l'ensemble des causes de décès au Canada. Le taux de mortalité par blessure non intentionnelle, que ce soit toutes causes confondues ou selon une causes déterminée (sauf pour les décès attribuables aux chutes), a été plus élevé chez les hommes que chez les femmes.

C'est dans les trois territoires que l'on a retrouvé les taux les plus élevés de mortalité, que l'on considère la mortalité globale ou celle par blessure non intentionnelle. De plus, la part de la mortalité 
TABLEAU 3

Taux de mortalité et ratio hommes-femmes pour la mortalité par blessure non intentionnelle, selon l'âge et les causes externes, Canada (à l'exclusion du Québec), 2001-2007

\begin{tabular}{|c|c|c|c|}
\hline & \multicolumn{2}{|c|}{ Taux de mortalité (pour 100 000) } & \multirow[t]{2}{*}{ Ratio hommes-femmes } \\
\hline & Hommes & Femmes & \\
\hline \multicolumn{4}{|l|}{ Groupe d'âge (ans) } \\
\hline $0-4$ & 7,5 & 5,3 & 1,42 \\
\hline $5-9$ & 4,3 & 3,2 & 1,34 \\
\hline $10-14$ & 6,2 & 3,5 & 1,77 \\
\hline $15-19$ & 29,0 & 12,0 & 2,42 \\
\hline $20-24$ & 37,7 & 12,1 & 3,12 \\
\hline $25-29$ & 32,0 & 8,5 & 3,76 \\
\hline $30-34$ & 28,5 & 8,6 & 3,31 \\
\hline $35-39$ & 30,4 & 9,8 & 3,10 \\
\hline $40-44$ & 33,1 & 10,6 & 3,12 \\
\hline $45-49$ & 34,8 & 11,9 & 2,92 \\
\hline $50-54$ & 33,3 & 11,5 & 2,90 \\
\hline $55-59$ & 33,3 & 12,7 & 2,62 \\
\hline $60-64$ & 35,3 & 14,4 & 2,45 \\
\hline $65-69$ & 42,8 & 21,0 & 2,04 \\
\hline $70-74$ & 56,9 & 32,3 & 1,76 \\
\hline $75-79$ & 98,0 & 61,4 & 1,60 \\
\hline $80-84$ & 187,2 & 122,5 & 1,53 \\
\hline 85-89 & 362,9 & 271,4 & 1,33 \\
\hline 90 et plus & 801,0 & 663,1 & 1,21 \\
\hline \multicolumn{4}{|l|}{ Causes externes } \\
\hline Collision de véhicules à moteur & 10,2 & 4,5 & 2,3 \\
\hline Piétons (en lien avec la circulation routière) & 1,3 & 0,8 & 1,6 \\
\hline Bicyclettes & 0,4 & 0,1 & 5,4 \\
\hline Navigation de plaisance & 0,2 & 0,0 & 10,4 \\
\hline Noyade & 1,5 & 0,4 & 4,3 \\
\hline Chutes & 7,7 & 7,9 & 1,0 \\
\hline Brûlures & 1,1 & 0,6 & 1,9 \\
\hline Suffocation & 1,6 & 1,1 & 1,4 \\
\hline Empoisonnement & 5,1 & 2,2 & 2,3 \\
\hline Autres & 7,9 & 5,6 & 1,4 \\
\hline
\end{tabular}

par blessure non intentionnelle dans la mortalité globale est presque deux fois plus élevée dans les trois territoires que dans les neuf provinces. Une étude populationnelle avec cas-témoins réalisée dans les Territoires du Nord-Ouest a révélé que les facteurs de risque de mourir de blessure étaient d'être de sexe masculin, d'être âgé de plus de 14 ans, d'habiter dans une collectivité éloignée, de vivre dans le Grand Nord et d'être autochtone ${ }^{11}$. Il y a une plus forte proportion d'Autochtones dans les territoires que dans le reste du Canada. Une étude réalisée auprès d'enfants albertains a femmes. Ce sont elles qui sont pour l'essentiel à l'origine de l'augmentation marquée de la mortalité par blessure non intentionnelle chez les jeunes et les jeunes adultes. Entre 2001 et 2007, la mortalité attribuable aux collisions de véhicules à moteur a peu varié d'une année sur l'autre chez les hommes, et a légèrement décliné chez les femmes. Les données ont toutefois fait ressortir des variations saisonnières, avec une augmentation significative du risque en été, encore plus marquée chez les hommes que chez les femmes. Durant les mois d'été, qui correspondent habituellement aux congés, les gens conduisent sur de plus longues distances, et les adolescents et les jeunes adultes ont sans doute davantage d'occasions de conduire des véhicules ou d'en être les passagers ${ }^{1}$; ils sont donc plus susceptibles d'être exposés aux facteurs de risque liés aux véhicules et à la circulation routière. Il est d'une importance cruciale de prévenir de façon efficace les collisions de véhicules à moteur pour réduire la mortalité par blessure non intentionnelle chez les jeunes et les jeunes adultes, en particulier les hommes.

Parmi les décès attribuables à une cause précise, seuls ceux dus aux chutes ont affiché une augmentation soutenue durant la période, et ils ont été légèrement plus fréquents chez les femmes que chez les hommes. Ils ont représenté environ un tiers de tous les décès par blessure non intentionnelle chez les adultes. Les chutes ont constitué la principale raison de la forte augmentation de la mortalité par blessure non intentionnelle touchant les personnes âgées. À l'échelle mondiale, les collisions de véhicules à moteur représentent $30 \%$ des décès par blessure non intentionnelle, les chutes simplement $11 \%{ }^{1}$; dans notre étude, ces proportions sont de respectivement $24 \%$ et $26 \%$ (figure 1). Quoique le processus de vieillissement et une faible densité minérale osseuse soient des facteurs généralement étroitement associés à la gravité de la blessure et aux conséquences d'une chute $^{8,14-19}$, on ignore s'ils sont à l'origine de l'augmentation soutenue de la mortalité que nous avons constatée. D’autres facteurs mériteraient de faire l'objet d'études plus poussées pour évaluer leur 
FIGURE 1

Répartition des décès par blessure non intentionnelle selon la cause au Canada (à l'exclusion du Québec), 2001-2007

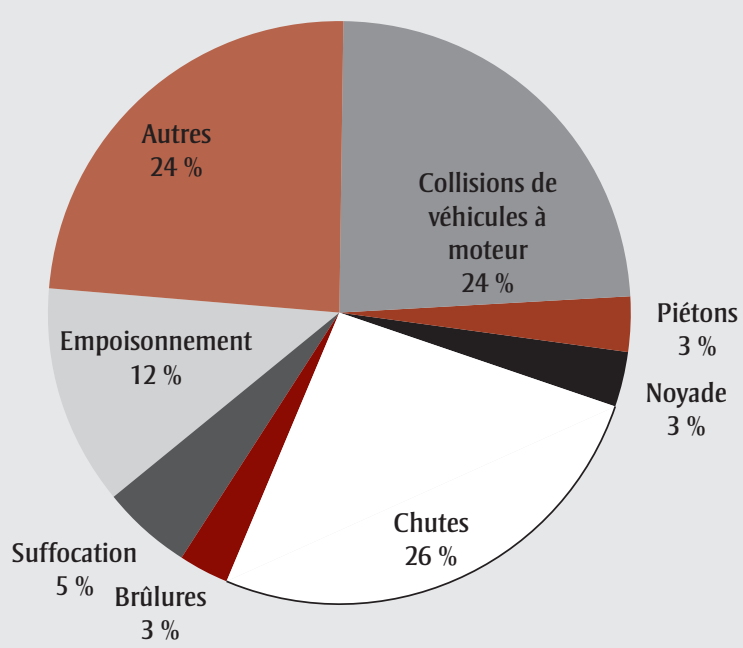

incidence possible sur la tendance à la hausse de la mortalité attribuable aux chutes dans la population canadienne : citons par exemple la prise de médicaments, en particulier par les personnes âgées $^{20}$, le surpoids et l'obésité ${ }^{20,21}$, le niveau d'activité physique ${ }^{23,24}$, l'utilisation de produits médicaux et les activités quotidiennes $^{25-28}$. Notre étude a également révélé l'existence d'une variation saisonnière de la mortalité attribuable aux chutes, avec un point culminant en novembre-décembre. Dans une étude effectuée aux États-Unis, on a constaté que certaines blessures consécutives à une chute étaient associées à la pose de décorations de Noël et autres activités connexes $^{29}$, ce qui est tout à fait plausible dans le contexte qui nous occupe. Les conditions météorologiques constituent vraisemblablement une autre cause importante in $^{30,31}$.

L'empoisonnement occupait le troisième rang des causes principales de décès par blessure non intentionnelle au Canada, et comptait pour $14 \%$ des décès par blessure non intentionnelle chez les hommes et pour $10 \%$ chez les femmes (données non présentées). L'empoisonnement non intentionnel peut être lié au travail ou à des agents courants comme les produits chimiques à usage domestique, les pesticides, les médicaments et les végétaux ${ }^{32-35}$. Les autres causes de décès par blessure non intentionnelle,

TABLEAU 4

Taux de mortalité globale et taux de mortalité par blessure non intentionnelle (pour 100 000), selon l'âge et le sexe, Canada (à l'exclusion du Québec), 2001-2007

\begin{tabular}{|c|c|c|c|c|c|c|c|c|c|}
\hline \multirow{2}{*}{$\begin{array}{l}\text { Groupe } \\
\text { d'âgé } \\
\text { (ans) }\end{array}$} & \multicolumn{3}{|c|}{ Les deux sexes } & \multicolumn{3}{|c|}{ Hommes } & \multicolumn{3}{|c|}{ Femmes } \\
\hline & $\begin{array}{c}\text { Toutes } \\
\text { causes, pour } \\
100000\end{array}$ & $\begin{array}{c}\text { Blessure non } \\
\text { intentionnelle, } \\
\text { pour } 100000\end{array}$ & $\begin{array}{c}\text { Blessure non } \\
\text { intentionnelle/ } \\
\text { Toutes causes (\%) }\end{array}$ & $\begin{array}{c}\text { Toutes } \\
\text { causes, pour } \\
100000\end{array}$ & $\begin{array}{c}\text { Blessure non } \\
\text { intentionnelle, } \\
\text { pour } 100000\end{array}$ & $\begin{array}{c}\text { Blessure non } \\
\text { intentionnelle/ } \\
\text { Toutes causes (\%) }\end{array}$ & $\begin{array}{c}\text { Toutes } \\
\text { causes, pour } \\
100000\end{array}$ & $\begin{array}{c}\text { Blessure non } \\
\text { intentionnelle, } \\
\text { pour } 100000\end{array}$ & $\begin{array}{c}\text { Blessure non } \\
\text { intentionnelle/ } \\
\text { Toutes causes (\%) }\end{array}$ \\
\hline $0-4$ & 124,4 & 6,43 & 5,2 & 135,4 & 7,5 & 5,5 & 112,9 & 5,3 & 4,7 \\
\hline $5-9$ & 11,7 & 3,73 & 31,9 & 13,0 & 4,3 & 33,1 & 10,3 & 3,2 & 30,8 \\
\hline $10-14$ & 14,3 & 4,89 & 34,2 & 16,2 & 6,2 & 38,3 & 12,3 & 3,5 & 28,2 \\
\hline $15-19$ & 45,9 & 20,78 & 45,2 & 62,1 & 29,0 & 46,7 & 28,8 & 12,0 & 41,8 \\
\hline $20-24$ & 60,0 & 25,19 & 42,0 & 85,8 & 37,7 & 43,9 & 33,1 & 12,1 & 36,6 \\
\hline $25-29$ & 58,5 & 20,35 & 34,8 & 82,6 & 32,0 & 38,7 & 34,0 & 8,5 & 25,0 \\
\hline $30-34$ & 69,6 & 18,60 & 26,7 & 92,1 & 28,5 & 30,9 & 46,8 & 8,6 & 18,4 \\
\hline $35-39$ & 95,9 & 20,12 & 21,0 & 122,1 & 30,4 & 24,9 & 69,4 & 9,8 & 14,1 \\
\hline $40-44$ & 140,6 & 21,91 & 15,6 & 174,9 & 33,1 & 18,9 & 106,0 & 10,6 & 10,0 \\
\hline $45-49$ & 221,8 & 23,35 & 10,5 & 272,9 & 34,8 & 12,8 & 170,8 & 11,9 & 7,0 \\
\hline $50-54$ & 349,8 & 22,34 & 6,4 & 431,0 & 33,3 & 7,7 & 269,7 & 11,5 & 4,3 \\
\hline $55-59$ & 545,1 & 22,92 & 4,2 & 674,1 & 33,3 & 4,9 & 417,8 & 12,7 & 3,0 \\
\hline $60-64$ & 881,9 & 24,67 & 2,8 & 1092,7 & 35,3 & 3,2 & 677,4 & 14,4 & 2,1 \\
\hline $65-69$ & 1406,4 & 31,51 & 2,2 & 1751,8 & 42,8 & 2,4 & 1082,9 & 21,0 & 1,9 \\
\hline $70-74$ & 2270,4 & 43,83 & 1,9 & 2850,2 & 56,9 & 2,0 & 1755,2 & 32,3 & 1,8 \\
\hline 75-79 & 3711,0 & 77,39 & 2,1 & 4684,2 & 98,0 & 2,1 & 2953,0 & 61,4 & 2,1 \\
\hline $80-84$ & 6157,3 & 147,78 & 2,4 & 7759,1 & 187,2 & 2,4 & 5130,4 & 122,5 & 2,4 \\
\hline $85-89$ & 10708,7 & 302,40 & 2,8 & 13173,0 & 362,9 & 2,8 & 9445,9 & 271,4 & 2,9 \\
\hline 90 et plus & 20590,7 & 700,09 & 3,4 & 23399,0 & 801,0 & 3,4 & 19562,0 & 663,1 & 3,4 \\
\hline
\end{tabular}


TABLEAU 5

Résultats de l'analyse de séries chronologiques (modèle autorégressif) pour les principales catégories de décès par blessure non intentionnelle (pour 100 000) chez les hommes

\begin{tabular}{|c|c|c|c|c|c|c|c|}
\hline \multirow[b]{2}{*}{ Variable } & \multicolumn{7}{|c|}{ Coefficient de régression (erreur-type) } \\
\hline & Véhicules à moteur & Chutes & Empoisonnement & Noyades & Piétons & Brûlures & Suffocation \\
\hline Année civile & $-0,0080(0,0072)$ & $0,0342(0,0039)^{* *}$ & $0,0090(0,0062)$ & $-0,0069(0,0023)^{*}$ & $0,0012(0,0016)$ & $-0,0028(0,0014)^{*}$ & $-0,0023(0,0021)$ \\
\hline \multicolumn{8}{|l|}{ Mois } \\
\hline $1-2$ & $-0,4768(0,0490)^{--}$ & $-0,0742(0,0280)^{*-}$ & $-0,0729(0,0325)^{\circ}$ & $-0,2976(0,0170)^{*}$ & $0,0011(0,0118)$ & $0,0311(0,0105)^{-*}$ & $-0,0124(0,0150)$ \\
\hline $3-4$ & $-0,4310(0,0490)^{--}$ & $-0,0936(0,0280)^{*}$ & $0,0354(0,0318)$ & $-0,2743(0,0169)^{*}$ & $-0,0173(0,0118)$ & $0,0310(0,0105)^{-*}$ & $-0,0116(0,0150)$ \\
\hline $5-6$ & $-0,1822(0,0484)^{*}$ & $-0,0701(0,0287)^{*}$ & $-0,0053(0,0281)$ & $-0,1435(0,0177)^{*}$ & $-0,0026(0,0121)$ & $0,0167(0,0111)$ & $-0,0227(0,0154)$ \\
\hline $9-10$ & $-0,1600(0,0484)^{-\prime}$ & $-0,0521(0,0287)$ & $-0,0310(0,0281)$ & $-0,2383(0,0177)^{-*}$ & $0,0336(0,0121)^{* *}$ & $0,0138(0,0111)$ & $-0,0306(0,0154)$ \\
\hline $11-12$ & $-0,2846(0,0490)^{*}$ & $0,0029(0,0280)$ & $-0,0243(0,0317)$ & $-0,2806(0,0169)^{*}$ & $0,0281(0,0118)^{*}$ & $0,0449(0,0105)^{-*}$ & $-0,0202(0,0150)$ \\
\hline
\end{tabular}

${ }^{*} p<0,05$

${ }^{* *} p<0,01$

comme la noyade et les brûlures, ont été moins fréquentes. Nos données ont montré, en conformité avec les résultats attendus, que les décès attribuables à la noyade sont survenus le plus souvent en été, et que les hommes et les enfants sont décédés par noyade dans une proportion plus élevée. La plupart des noyades sont associées à des activités de loisirs. Nos données ont également fait ressortir une augmentation de la mortalité attribuable aux brûlures en hiver. Aucune variation saisonnière des décès par suffocation n'a été observée.

\section{Limites}

L'enregistrement des décès étant obligatoire au Canada, les données de l'état civil sont relativement complètes. C'est toutefois la cause initiale de décès - définie comme étant la maladie ou la blessure à l'origine de la séquence des événements ayant conduit directement au décès - qui est prise en considération. Or une personne ne décédant pas forcément immédiatement après avoir subi une blessure, c'est plutôt le code correspondant à une affection subséquente (p. ex. une insuffisance cardiaque) qui est susceptible d'être inscrit comme cause principale de décès. La blessure reste alors étroitement liée au décès sans pour autant être considérée comme sa cause initiale. Cette méthode d'enregistrement des décès repose sur le jugement des médecins légistes ou des coroners. Les diagnostics secondaires étant exclus, le fardeau réel de la mortalité par blessure non intentionnelle risque d'être de ce fait sous-estimé. De plus, les erreurs de codage et de saisie des données peuvent engendrer une classification erronée de l'information sur la

FIGURE 2

Taux mensuel de mortalité normalisé attribuable à différentes catégories de blessures non intentionnelles chez les hommes au Canada (à l'exclusion du Québec), 2001-2007

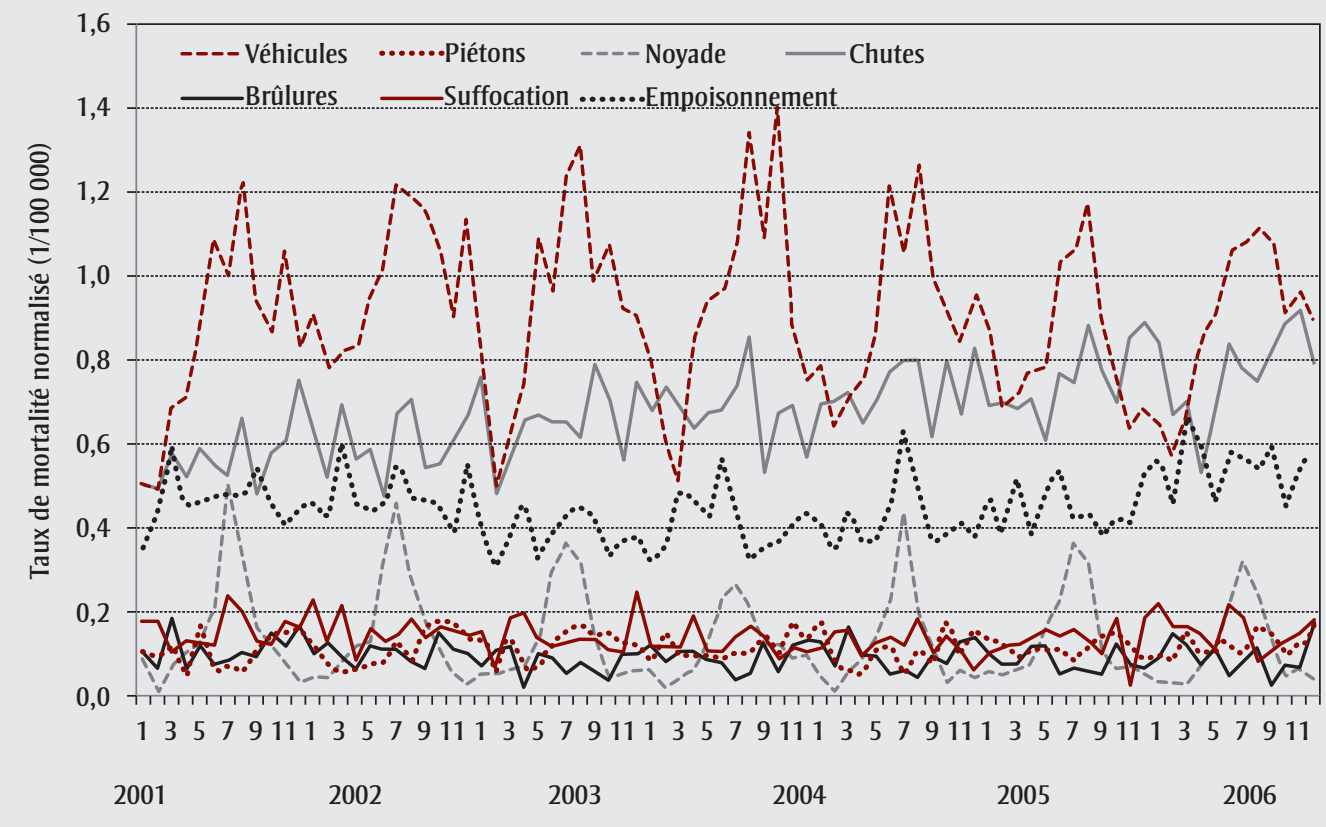


TABLEAU 6

Résultats de l'analyse de séries chronologiques (modèle autorégressif) pour les principales catégories de décès par blessure non intentionnelle (pour 100 000) chez les femmes

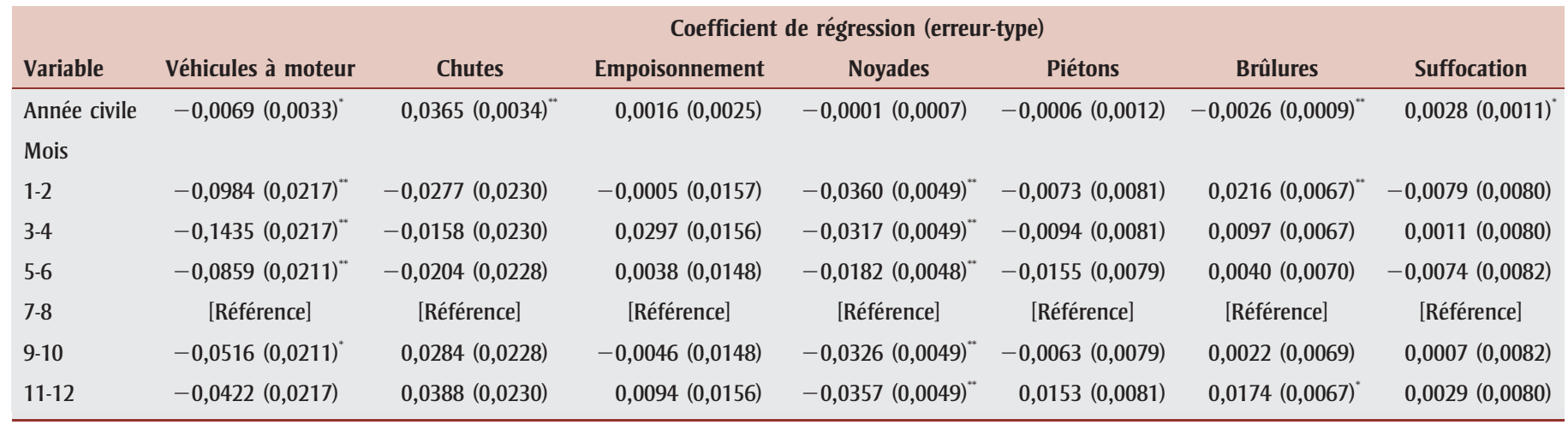

${ }^{*} p<0,05$

${ }^{* *} p<0,01$

cause du décès et sur les causes externes des blessures. Enfin, la période étudiée (2001-2007) est relativement courte.

\section{Conclusion}

Entre 2001 et 2007, la mortalité par blessure non intentionnelle a peu évolué, alors que la mortalité globale déclinait régulièrement au Canada. C'est dans les trois territoires que l'on a observé la mortalité par blessure non intentionnelle la plus élevée, tant en valeur absolue qu'en proportion de la mortalité globale. Les collisions de véhicules à moteur et les chutes ont été les principales causes de décès par blessure non intentionnelle. La mortalité attribuable aux chutes a été la seule catégorie de mortalité par blessure non intentionnelle ayant affiché une augmentation annuelle. Les décès attribuables aux chutes ont été plus fréquents chez les femmes, tandis que les autres types de décès par blessure non intentionnelle ont été plus répandus chez les hommes, les taux de mortalité associés restant stables sur la période. Nous avons observé des variations saisonnières pour certains types de décès par blessure non intentionnelle : risques plus élevés de décès attribuables en été aux collisions de véhicules à moteur et aux noyades, en hiver aux chutes et aux brûlures et incendies. Étant donnée la part croissante de la mortalité par blessure non intentionnelle dans la mortalité toutes causes confondues, et la tendance à la hausse de la mortalité attribuable aux chutes, il nous apparaît important d'intensifier les recherches sur la détermination

FIGURE 3

Taux mensuel de mortalité normalisé attribuable à différentes catégories de blessures non intentionnelles chez les femmes au Canada (à l'exclusion du Québec), 2001-2007

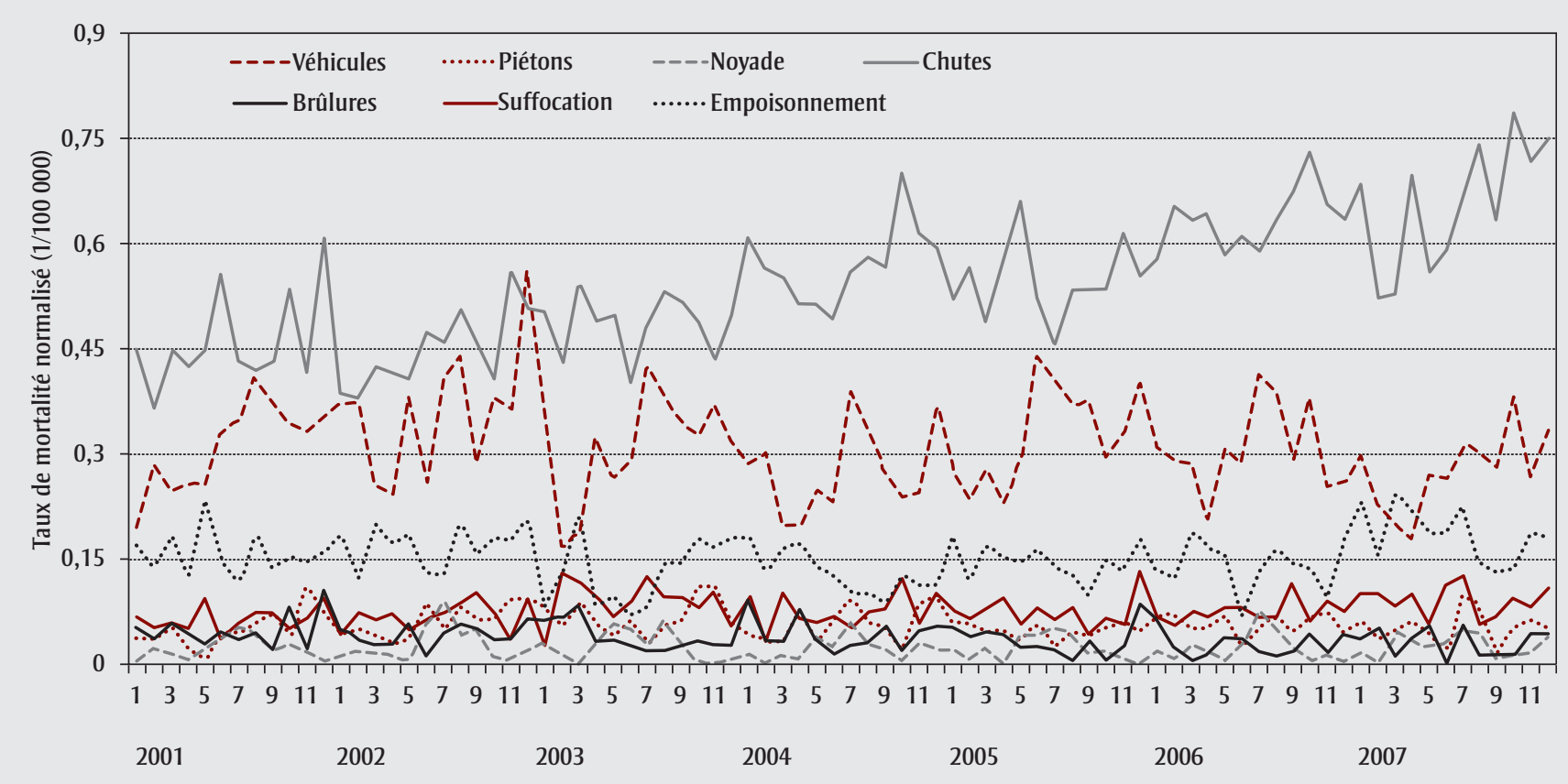


des facteurs de risque et les interventions efficaces.

\section{Remerciements}

Nous adressons nos remerciements aux personnes suivantes pour leur collaboration au projet "Évaluation des risques et des blessures liés aux produits de consommation »: $\mathrm{D}^{\mathrm{r}}$ Howard Morrison, superviseur principal et directeur du projet; M. Doug Hopkins, gestionnaire du projet; $\mathrm{M}^{\mathrm{me}}$ Caroline Da Silva, responsable de la gestion et de la coordination du projet.

\section{Références}

1. Chandran A, Hyder AA, Peek-Asa C. The global burden of unintentional injuries and an agenda for progress. Epidemiol Rev. 2010;32:110-20.

2. Krug EG, Sharma GK, Lozano R. The global burden of injuries. Am J Public Health. 2000;90:523-6.

3. Peden MM, McGee K, Krug E (dir.). Injury : a leading cause of the global burden of disease, 2000. Geneva (CH) : World Health Organization; 2002.

4. Violence, injuries and disability biennial report, 2006-2007. Geneva (CH) : World Health Organization; 2008.

5. Mulholland E; Comité consultatif sur le fardeau économique des blessures au Canada. Le fardeau économique des blessures au Canada. Toronto (ON) : SAUVEQUI-PENSE; 2009.

6. Harrison JE. Injury classification: balancing continuity and utility. Inj Control Safety Promot. 2000;7:51-63.

7. Curry AE, Hafetz J, Kallan MJ, Winston FK, Durbin DR. Prevalence of teen driver errors leading to serious motor vehicle crashes. Accid Anal Prev. 2011;43:1285-90.

8. Kannus P, Parkkari J, Niemi S, Palvanen M. Fall-induced deaths among elderly people. Am J Public Health. 2005;95:422-4.
9. Statistique Canada. Estimations démographiques annuelles : Canada, provinces et territoires [Internet]. Ottawa (Ont.) : sept. 2011. [Statistique Canada, $n^{\circ}$ 91-215-X au catalogue]. Consultable en ligne à la page : http://www.statcan.gc.ca/pub/91-215-x/ 91-215-x2011000-fra.htm

10. Statistique Canada. Statistiques démographiques annuelles, 2005. Ottawa (Ont) : 2006. [Statistique Canada, n CS91-213/ 2005 au catalogue].

11. Mo D. Injury mortality risk assessment and targeting the subpopulations for prevention in the Northwest Territories, Canada. Int $\mathrm{J}$ Circumpolar Health. 2001;60:391-9.

12. Harrop AR, Brant RF, Ghali WA, Macarthur C. Injury mortality rates in Native and nonNative children : a population-based study. Public Health Rep. 2007;122:339-46.

13. Stevenson MR, Wallace LJ, Harrison J, Moller J, Smith RJ. At risk in two worlds : injury mortality among indigenous people in the US and Australia, 1990-92. Aust N Z J Public Health. 1998;22:641-4.

14. Rauh MJ, Nichols JF, Barrack MT. Relationships among injury and disordered eating, menstrual dysfunction, and low bone mineral density in high school athletes: a prospective study. J Athl Train. 2010;45:243-52.

15. Sakai A, Oshige T, Zenke Y, Yamanaka Y, Otsuka H, Nakamura T. Shorter unipedal standing time and lower bone mineral density in women with distal radius fractures. Osteoporos Int. 2009;21:733-9.

16. Sone T. [Increase in bone mineral density and its effect on fracture risk]. Clin Calcium. 2005;15:625-9.

17. Hartholt KA, Stevens JA, Polinder S, van der Cammen TJ, Patka P. Increase in fallrelated hospitalizations in the United States, 2001-2008. J Trauma. 2011;71: 255-8.

18. Hartholt KA, van Beeck EF, Polinder S, van der Velde N, van Lieshout EM, Panneman MJ collab. Societal consequences of falls in the older population : injuries, healthcare costs, and long-term reduced quality of life. J Trauma. 2011;71:748-53.
19. Melton LJ 3rd, Riggs BL. Risk factors for injury after a fall. Clin Geriatr Med. 1985; 1:525-39.

20. Hartikainen S, Lonnroos E, Louhivuori K. Medication as a risk factor for falls : critical systematic review. J Gerontol A Biol Sci Med Sci. 2007;62:1172-81.

21. Finkelstein EA, Chen $H$, Prabhu $M$, Trogdon JG, Corso PS. The relationship between obesity and injuries among U.S. adults. Am J Health Promot. 2007;21: 460-8.

22. Hu HY, Chou YJ, Chou P, Chen LK, Huang N. Association between obesity and injury among Taiwanese adults. Int $\mathrm{J}$ Obes (Lond). 2009;33:878-84.

23. Appleby PN, Allen NE, Roddam AW, Key TJ. Physical activity and fracture risk : a prospective study of 1898 incident fractures among 34,696 British men and women. J Bone Miner Metab. 2008;26:191-8.

24. Gill DP, Zou GY, Jones GR, Speechley M. Injurious falls are associated with lower household but higher recreational physical activities in community-dwelling older male veterans. Gerontology. 2008;54:10615 .

25. Belechri M, Petridou E, Trichopoulos D. Bunk versus conventional beds: a comparative assessment of fall injury risk. J Epidemiol Community Health. 2002;56: 413-7.

26. Dellinger AM, Boyd RM, Haileyesus T. Fall injuries in older adults from an unusual source: entering and exiting a vehicle. J Am Geriatr Soc. 2008;56:609-14.

27. O’Neil J, Steele GK, Huisingh C, Smith GA. Escalator-related injuries among older adults in the United States, 1991-2005. Accid Anal Prev. 2008;40:527-33.

28. Stevens JA, Thomas K, Teh L, Greenspan AI. Unintentional fall injuries associated with walkers and canes in older adults treated in U.S. emergency departments. J Am Geriatr Soc. 2009;57:1464-9.

29. Centers for Disease Control and Prevention (CDC). Fall-related injuries during the holiday season-United States, 2000-2003. MMWR Morb Mortal Wkly Rep. 2004;53: 1127-9. 
30. Jacobsen SJ, Sargent DJ, Atkinson EJ, O'Fallon WM, Melton LJ, 3rd. Contribution of weather to the seasonality of distal forearm fractures : a population-based study in Rochester, Minnesota. Osteoporos Int. 1999;9:254-9.

31. Wareham K, Johansen A, Stone MD, Saunders J, Jones S, Lyons RA. Seasonal variation in the incidence of wrist and forearm fractures, and its consequences. Injury. 2003;34:219-22.

32. Miech R, Koester S, Dorsey-Holliman B. Increasing US mortality due to accidental poisoning : the role of the baby boom cohort. Addiction. 2010;106:806-15.

33. Yoon YH, Stinson FS, Yi HY, Dufour MC. Accidental alcohol poisoning mortality in the United States, 1996-1998. Alcohol Res Health. 2003;27:110-8.

34. Kivisto JE, Parkkari J, Mattila VM, Hoppu K. Poisoning deaths among Finnish children from 1969 to 2003. Acta Paediatr. 2009;98:1661-6.

35. Meyer S, Eddleston M, Bailey B, Desel H, Gottschling S, Gortner L. Unintentional household poisoning in children. Klin Padiatr. 2007;219:254-70. 